\title{
Isolation of extracellular vesicles: Determining the correct approach (Review)
}

\author{
RAFAL SZATANEK, JAREK BARAN, MACIEJ SIEDLAR and MONIKA BAJ-KRZYWORZEKA
}

Department of Clinical Immunology and Transplantology, Jagiellonian University Medical College, 30-663 Krakow, Poland

Received February 27, 2015; Accepted April 22, 2015

DOI: $10.3892 / \mathrm{ijmm} .2015 .2194$

\begin{abstract}
The discovery of extracellular vesicles (EVs) has revised the interpretation of intercellular communication. It is now well established that EVs play a significant role in coagulation, inflammation, cancer and stem cell renewal and expansion. Their release presents an intriguing, transporting/trafficking network of biologically active molecules, which are able to reach and modulate the function/behavior of the target cells in a variety of ways. Moreover, the presence of EVs in various body fluids points to their potential for use as biomarkers and prognostic indicators in the surveillance/monitoring of a variety of diseases. Although vast knowledge on the subject of EVs has accumulated over the years, there are still fundamental issues associated with the correct approach for their isolation. This review comprises the knowledge on $\mathrm{EV}$ isolation techniques that are currently available. The aim of this reveiw was to make both experienced researchers and newcomers to the field aware that different types of EVs require unique isolation approaches. The realization of this 'uniqueness' is the first step in the right direction for the complete assessment of EVs.
\end{abstract}

\section{Contents}

1. Introduction

2. Concept of EV isolation

3. Cell culture medium

4. Body fluids

5. Storage and isolation conditions

6. Isolation

7. Polymeric precipitation

8. Biological activities of EVs and associated challenges

9. Conclusion

Correspondence to: Dr Monika Baj-Krzyworzeka, Department of Clinical Immunology and Transplatology, Jagiellonian University Medical College, ul. Wielicka 265, 30-663 Krakow, Poland E-mail: mibaj@cyf-kr.edu.pl

Key words: extracellular vesicles, exosomes, microvesicles, differential centrifugation

\section{Introduction}

Intercellular communication in a multicellular organism is essential for its proper functionality. Traditionally, soluble factors released by different types of cells were considered to serve such a role in a localized environment (1). With the emergence and discovery of small membrane sacs, later termed as extracellular vesicles (EVs), that are released by various cell types, the concept of 'cell-to-cell talk' has been re-evaluated $(2,3)$. It is now widely accepted that EVs have a substantial contribution to intercellular communication by shuttling bioactive molecules (transmembrane receptors, mRNAs, miRNAs and signaling molecules) that are able to modulate the extracellular environment (4-9). The term EV implies to all shed membrane vesicles, which can be further classified on the basis of their size, origin and their cargo (10). In general, however, EVs have been divided into two groups, exosomes and microvesicles $(5,10,11)$. Exosomes are formed through invagination into endosomes to form multivesicular bodies (MVBs) and are thought to be somewhat unique in their protein and lipid composition (10). Due to their endosomal origin, exosomes contain membrane transport and fusion proteins (GTPases, Annexins and flotillin), tetraspannins (CD9, CD63, CD81 and CD82), heat shock proteins (Hsc70 and Hsp90), proteins involved in multivesicular body biogenesis (Alix and TSG101), as well as lipid-related proteins and phospholipases $(10,12,13)$, although other markers have also been associated with them (14). On the other hand, microvesicles are particles shed from the plasma membrane following stimulation and are often a hallmark of cell apoptosis (15-17). Upon cell activation, as a result of stimuli, such as shear stress or cytokine/endotoxin release, the cytosolic calcium concentration increases, leading to the activation of a number of enzymes (calpains, gelsolins, scramblases and kinases) (16). This, in turn, leads to the inhibition of enzymes, such as translocases and phosphatases, resulting in cytoskeletal reorganization, the loss of membrane asymmetry and membrane blebbing, which causes microvesicle formation and release $(16,18)$. Microvesicles have a rich phospholipid bilayer consisting of phosphatidyl serine on the outer leaflet and their membrane proteins reflect those of the cell they originate from $(15,17,18)$.

\section{Concept of EV isolation}

The concept of EV isolation begins with the nature of the sample itself. First of all, one has to realize that different samples present 
unique obstacles when it comes to EV isolation, which is greatly related to the origin of the EV. Thus, with respect to the source, EVs can be divided into two groups: i) those isolated from cell culture media, and ii) those isolated from body fluids (plasma, urine, spinal fluid, saliva, etc.).

\section{Cell culture medium}

When isolating EVs from conditioned cell culture media one has to consider the presence of an additional, 'artificial' EV source, namely fetal bovine serum (FBS) or any other supplement for that matter, which is routinely added to cell cultures $(19,20)$. Our results obtained by nanoparticle tracking analysis (NTA) showed freshly prepared cell culture medium supplemented with FBS to have a substantial particle population (mostly $<100 \mathrm{~nm}$ in size) already present before the actual use (Fig. 1). This observation alone clearly demonstrates that the downstream EV isolation from conditioned cell culture media carries along the risk of obtaining, apart from proper EVs, also those from FBS, which obviously, obscures the final results. This observation is in accordance with observations of other groups, which have also shown FBS to contain vesicles that may be later isolated along the actual EV (19-21). To counter that problem, many groups apply filters to remove EVs from the media/FBS or use a long ultracentrifugation step. In an elegant study, Lötvall et al showed that the removal of FBS-originated EVs is critical for further downstream experiments, since these vesicles are capable of inducing effects similar to those of EVs isolated from the actual cell line culture media (21). Thus, it has been proposed that a $16 \mathrm{~h}$-ultracentrifugation step at $100,000 \mathrm{x} \mathrm{g}$ or greater for FBS is absolutely obligatory for a complete FBS vesicle depletion, since shorter centrifugation steps are insufficient $(19,21)$. An alternative may be the use of an exosome-free FBS, which is already commercially available, but is still rather expensive. Some also postulate the use of bovine serum albumin (BSA) instead of the standard FBS (20).

Another major issue involving EV isolation from conditioned cell culture media regards the culture medium itself. Our NTA results revealed that even a newly opened cell culture medium contains a trace of particles that resemble EVs sizewise (Fig. 1). The presence of these background particles itself puts tremendous strain on the integrity of the final EV isolation results. Similar results were obtained by Jeppesen $e t$ al, who compared two different cell culture media and showed that one type had more particle background than the other (19). Moreover, the storage temperature also seems to have an effect on the occurrence of the particles in a cell culture medium. A cell culture medium stored at room temperature showed more background particles than the one stored at $4^{\circ} \mathrm{C}$ as observed by NTA, which was even more apparent with time passage (own data). It has been suggested that in such cases, ultracentrifugation for an extended period of time ( $16 \mathrm{~h}$ or longer) is necessary for the removal of particle background from the cell culture media (19).

There seems to be a strong demand for establishing a uniform protocol for EV isolation, particularly for EVs which are being isolated from cell culture media; however, this may not be so simple. Studies have shown that different cell lines generate unique EVs, suggesting that they should be considered individually with respect to the isolation approach. In

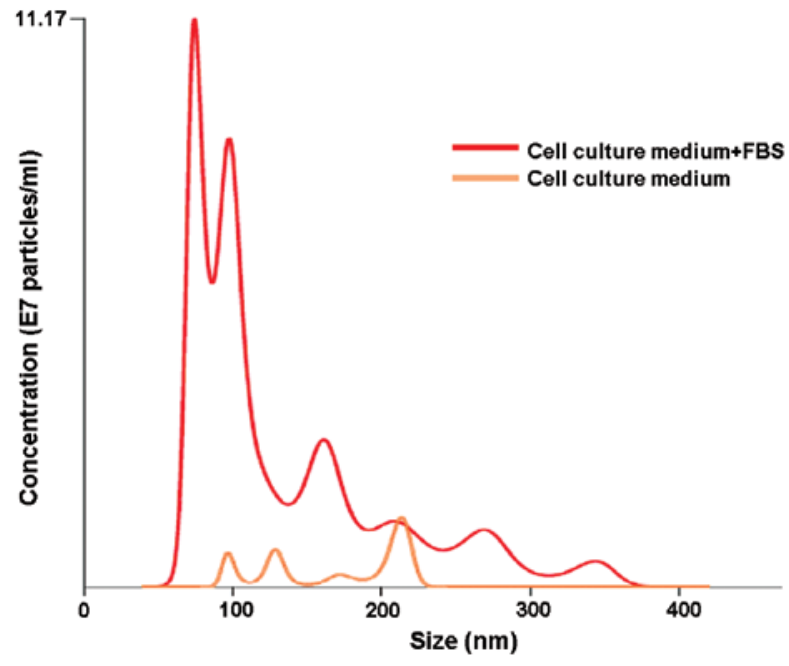

Figure 1. Size distribution of particles obtained by nanoparticle tracking analysis (NTA) from cell culture medium sample supplemented with fetal bovine serum (FBS) and from freshly opened cell culture medium.

their study, Jeppesen et al showed that the optimal isolation conditions for EVs obtained from embryonic kidney HEK293 and bladder carcinoma FL3 cells differed from each other (19). Their study indicated that the optimal vesicle-to-protein yield for HEK293 cells was obtained at 67,000 x g, while that for FL3 cells was at 100,000 x g. Furthermore, it was demonstrated that specific g-force/k-factor usage during differential centrifugation greatly influences the purity and yield of exosomes (19).

\section{Body fluids}

EV isolation from body fluids seems to be even more complex. Although the problem of the FBS vesicle presence obviously does not apply in this case, there are other factors (lipoproteins, DNA, RNA, protein aggregates and microbes) that need to be addressed for proper isolation (22-26). The majority of the available data concerns the overall EV population isolated from blood samples; however, there are attempts to 'select out' from that EV pool a certain type of EV depending on its origin (i.e., platelet, epithelial, leukocyte and tumor). Again, a careful approach needs to be undertaken for the isolation of the 'wanted' EV fraction(s). For example, if the isolation of leukocyte-origin EV is desired, the sample needs to be depleted of platelets. For that to be accomplished, platelets need to be centrifuged at a certain speed and under appropriate conditions (discussed in further detail below) to avoid platelet activation and, consequently, the generation of EVs of platelet origin $(27,28)$. It has to be kept in mind that a blood sample (or any other body fluid sample for that matter) is a source of many types of EVs. Realizing the complexity of such samples puts another perspective on the $\mathrm{EV}$ fraction isolation approaches.

\section{Storage and isolation conditions}

There are no strictly defined conditions for storing/isolating EVs. One exception, however, are platelet-derived EVs, where firm guidelines for their proper acquisition have been laid down by the Vascular Biology group of the Scientific and Standardization Committee of the International Society 
on Thrombosis and Hemostasis over a decade ago (27-29). Although some basic framework has been established for other EVs, many groups have still independently developed their own storage/isolation protocols that are suitable to their individual laboratory settings (30). There is a general understanding that freshly acquired and processed samples guarantee the best EV yield; however, that is, for the most part, not possible $(30,31)$.

Body fluid samples (blood, serum, plasma, urine and tumor ascites) are collected/stored in a number of ways. For example, there are studies demonstrating that blood samples collected on different anticoagulants, when processed further downstream, exhibit different EV yields $(27,28)$. It has been shown that platelet and endothelial EV counts were substantially lower in blood samples collected in citrate or EDTA than in the ones collected in protease inhibitors, either hirudin and soybean trypsin inhibitor or heparin $(27-29,32)$. Some groups follow strict protocols and process their blood samples within $1 \mathrm{~h}$ after collection (33). Others believe that a fasting period (of up to $12 \mathrm{~h}$ ) prior to the sample collection is necessary for proper EV acquisition $(27,28,34)$. On the other hand, others have indicated that storing blood samples, or any other body fluid samples for that matter, at $4^{\circ} \mathrm{C}$ for up to 5 days does not affect the final EV yield (20). Observations have also been made that the EV diameter significantly decreases with the duration of the storage period (e.g., within 2 days) and with the increasing temperature during storage (from $4-37^{\circ} \mathrm{C}$ ) $(35)$. Freezing and thawing cycles are also critical. Most groups are in agreement that multiple freezing-thawing cycles of a sample affect EV characteristics/concentration, although some suggest that repeating these cycles for up to 10 times has no influence on the size and composition of EVs to any significant degree (29-32,35). Moreover, the thawing conditions alone seem to play a crucial role in EV recovery. It has been observed that EV samples thawed on ice showed a lower EV recovery compared to the ones thawed at room temperature or $37^{\circ} \mathrm{C} \mathrm{(36).}$ To resolve this issue, aliquots of once obtained EV stocks are stored at -70 to $-80^{\circ} \mathrm{C}$ until use for up to a year, which then undergo only one freezing/thawing cycle (20,34). Zhou et al reported that the storage of EVs isolated from urine at $-20^{\circ} \mathrm{C}$ resulted in a major loss of $\mathrm{EVs}$, while storage at $-80^{\circ} \mathrm{C}$ had no effect on the EV recovery (37).

In our opinion, the general rule for storage and isolation conditions should be 'the sooner, the better'. The starting sample should be handled rapidly after collection, avoiding extensive waiting periods between further processing stages (i.e., centrifugation steps). In blood/plasma samples, all the necessary precautions (i.e., processing temperature, upright sample position for transport, no agitation) should be undertaken to avoid platelet activation, and thus potential platelet-derived EV generation. Moreover, once isolated, EV aliquots should be prepared, and they should undergo only one freezing/thawing cycle at an appropriate temperature to ensure optimal EV recovery.

\section{Isolation}

There are basically three major methodologies used for EV purification/isolation that serve as the backbone for potential method variations: i) differential centrifugation/ultracentrifugation with/without a sucrose gradient/cushion; ii) adsorption to magnetic/non-magnetic microbeads; and iii) size exclusion chromatography.

Differential centrifugation/ultracentrifugation. This is probably the most commonly used method for EV isolation/purification $(20,30,38)$. As with other methods, there are variations depending on the laboratory setting; however, for the most part, the protocols follow the scheme that was put forward in the study by Raposo et al, who purified exosomes from the conditioned culture media of transformed human $\mathrm{B}$ cell lines (38). The protocol involves a number of sequential centrifugation steps at different centrifugal forces $(\mathrm{g})$ whose purpose is to remove unwanted components from the actual exosomes. The first three steps of the protocol are designed to remove intact cells, dead cells or cell debris using three different centrifugal forces, that is $300 \mathrm{x} \mathrm{g}$ for $10 \mathrm{~min}, 2,000 \mathrm{xg}$ for $10 \mathrm{~min}$ and $10,000 \mathrm{x} \mathrm{g}$ for $30 \mathrm{~min}$, respectively. After each centrifugation, the supernatant is transferred into a new test tube while the generated pellets are being discarded. After the $10,000 \mathrm{x} \mathrm{g}$ spin, the supernatant is then subjected to a final ultracentrifugation at $100,000 \times \mathrm{g}$ for $70 \mathrm{~min}$. The outcome of this step is an exosome pellet that can be used for further studies. It should be also noted that all the centrifugation steps are being carried out at $4^{\circ} \mathrm{C}$. The basic scheme for $\mathrm{EV}$ isolation is presented in Fig. 2.

Although the protocol presented in the study by Raposo et al (38) served as the backbone for others, it focused only on the purification of exosomes, a portion of the EV population with a size of $<100 \mathrm{~nm}$, and their isolation from conditioned cell culture media. With the discovery of EVs being present in all types of body fluids, there is an increasing need to adopt the protocol for appropriate samples with the incorporation of a step(s) enabling the isolation of EVs with a larger size $(>100 \mathrm{~nm})$. The EVs that can be obtained from a body fluid sample are divided into four distinct $\mathrm{EV}$ populations: exosomes, microvesicles, apoptotic bodies and microsomes (19). Exosomes (40-100 nm), the most extensively studied EV population, are usually isolated by centrifugation at 100,000 $200,000 \times \mathrm{g}(20,39)$, whereas microvesicles $(100-1,000 \mathrm{~nm})$ are isolated by centrifugation at $10,000-20,000 \times \mathrm{g}(30,40)$. Apoptotic bodies $(50-5,000 \mathrm{~nm})$ are obtained at a $\mathrm{g}$-force of approximately 2,000 x g (19), whereas microsomes are $80-120 \mathrm{~nm}$ in size and their isolation/identification needs to be confirmed by additional means $(19,41,42)$. For the isolation of EVs from plasma, lymph fluid, urine, bronchiolar lavage fluid and tumor ascites, Théry et al proposed diluting the samples with an equal volume of PBS before further processing due to the viscosity of the respective fluids (20). Moreover, due to the complexity of the viscous fluid samples the time and centrifugation speeds have been increased/adapted to obtain the appropriate EV populations. The major difference compared to the protocol presented in the study by Raposo et al (38) was made at the beginning of the modified protocol. Here, after the initial centrifugation step at $300 \mathrm{x}$ g, another additional centrifugation at 2,000-3,000 $\mathrm{x}$ for up to $30 \mathrm{~min}$ is performed, followed by the $12,000-15,000 \mathrm{x} g$ step for up to $1 \mathrm{~h}$. The purpose of the first step is to deplete the sample of cells/cellular debris. The second step enables the acquiring of apoptotic bodies, whereas the third one eliminates platelets/platelet EVs (plasma samples) and/or isolates microvesicles. Some groups at this 


\section{Conditioned medium/bodily fluid (dilute with equal volume of PBS)}

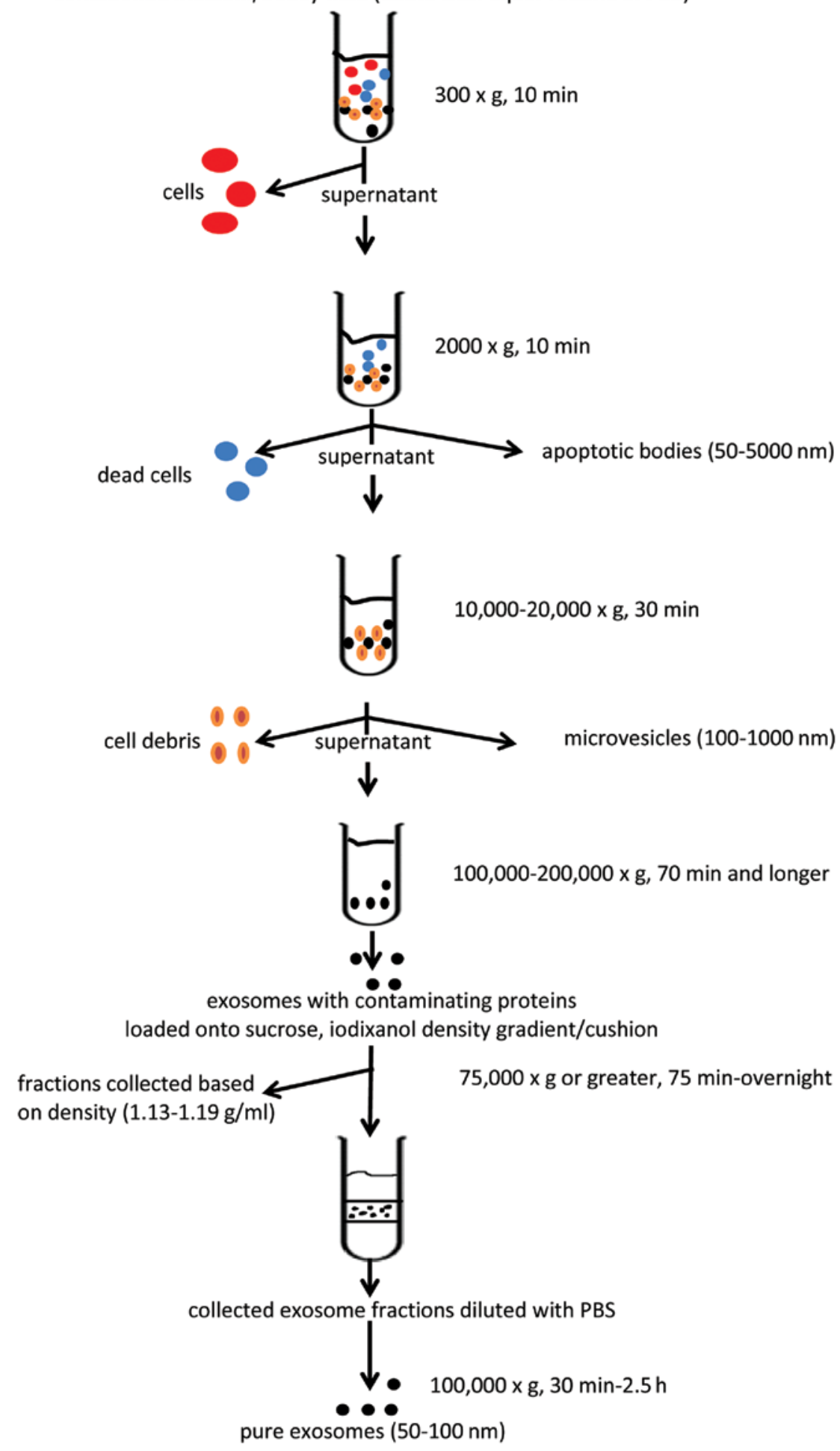

Figure 2. Differential centrifugation scheme, including a sucrose, iodixanol gradient/cushion step.

point check whether platelets/platelet EV have been removed from the centrifuged samples by anti-CD41, -CD61 monoclonal antibody staining and flow cytometry analysis (40). Only platelet-free plasma (PFP) and platelet EV-free plasma samples undergo further processing. It should be noted that, although some groups recommend centrifugation at $4^{\circ} \mathrm{C}$ at all times, that should not be the case when dealing with plasma samples. The processing of these samples at this temperature leads to platelet activation, which in turn causes the generation of platelet EVs that may become difficult to remove downstream.

A variation of the $\mathrm{EV}$ isolation method by centrifugation that has been adopted by some groups includes an additional sucrose gradient/cushion step (43-45). Some argue that during $\mathrm{EV}$ isolation by centrifugation, aggregates of large proteins and/ or proteins that were non-specifically associated with EVs are also being sedimented $(20,45)$. A sucrose gradient $(20-60 \%) /$ 
cushion (30\% sucrose) step incorporated into the centrifugation protocol supposedly eliminates this contamination, and the resulting EV population seems to be of a greater purity. The diluted (with PBS) EV suspension obtained by the protocol discussed above is gently loaded onto the Tris/sucrose $/ \mathrm{D}_{2} \mathrm{O}$ solution containing tube and ultracentrifuged for a period of time (75 min up to overnight at $75,000 \mathrm{x}$ g or greater) $(20,44-$ 47). Following centrifugation, the EV fractions are collected based on their density, that ranges between 1.13-1.19 $\mathrm{g} / \mathrm{ml}$, diluted with PBS and centrifuged again for $30 \mathrm{~min}-2.5 \mathrm{~h}$, at $100,000 \times \mathrm{g}(20,44-47)$. Although the incorporation of the density gradient step into the $\mathrm{EV}$ isolation protocol has been designed to purify and isolate specific EV fractions, it has been recently reported that certain high-density lipoproteins (HDLs) can also be isolated using this method (48). An alternative to these problems may be the OptiPrep velocity gradient. In this method, 5-40\% iodixanol gradient is used instead of sucrose, showing, reportedly, an improved separation of EVs from viral particles and small apoptotic bodies (47). Moreover, unlike sucrose, iodixanol is capable of forming iso-osmotic solutions at all densities, thus better preserving the size of the vesicles in the gradient (46).

Immunoaffinity isolation. Another method used for EV isolation involves microbeads, usually magnetic, that are coated with an antibody that recognizes certain markers present on the EV surface. This technology can be used for EV isolation from either cell culture media or body fluids. After mixing the EV sample with the antibody-coated microbeads, a magnetic force is applied (i.e., to a column, microplate) which retains the EV-covered microbeads, while the rest of the sample is discarded (www.systembio.com/exosomes). Next, the microbeads with attached EV are eluted using appropriate buffers and used for further analysis. The advantage of this isolation method is its ability to select a specific EV population based on a marker expression regardless of its size. Many groups put in a lot of effort into selecting an EV fraction (i.e., exosomes and microvesicles) based on EV size, but not on the surface marker profile. Although this approach may result in obtaining valuable information on such EV fractions, it limits, however, the overall scope on the impact that the whole EV population, regardless of size, may exert on the surroundings. This is particularly evident in an in vivo setting where different types of cells may generate EVs of different sizes, but still carry the same surface marker (10). Another important advantage is the ability of coupling this method with other methods (i.e., flow cytometry, western blotting and 'real-time' PCR) to characterize even further the already selected/specific EV fraction. At the same time, however, it needs to be pointed out that the beads, due to their physical binding surface area, can only bind a certain number of EVs, which, in turn, can lead to a substantial population of EVs being still left out from the sample or lost during the purification process.

An alternative to magnetic microbeads are surfactant-free latex beads typically made of polystyrene (49), which are stabilized against aggregation by covalently linked charge groups (sulfate, carboxyl, amidine, carboxyl/sulfate, aldehyde/sulfate, chloromethyl and aldehyde/amidine) and have $95 \%$ of their surface available for passive adsorption of proteins (www.Invitrogen.com). They can be either hydrophobic or hydrophilic and can covalently bind EVs regardless of their size or surface marker composition. Due to this feature, the bound EVs can be further characterized by, for example, flow cytometry using multi-antibody staining, thus providing valuable information on the EVs. Although this method can lead to the acquisition of informative data on EVs, their elution from the beads is difficult. This is a major disadvantage to this method, since the bound EVs cannot be used in downstream experiments.

Size exclusion chromatography. This method is usually coupled with a low-speed centrifugation step that allows the removal of larger objects from the sample (cells, cellular debris, organelles, etc.) that is followed by a filtration step $(0.8$ and $0.2 \mu \mathrm{m}$ pore size filter) to pre-concentrate the EVs. The filtered EV sample is then subjected to size exclusion chromatography (usually gel filtration column) where small volume fractions $(1 \mathrm{ml})$ of the filtrate are collected and ultracentrifuged (100,000 x g, $1 \mathrm{~h}$ and longer) to pellet down the EVs (50-52). The principal behind this technique is that particles in a sample, depending on their size, will move through the filtration column at different rates. Thus, larger particles will elute more rapidly, while the smaller ones more slowly, due to their ability to penetrate the stationary phase (gel) of the column. In theory, the obtained eluted fraction at a certain time should contain a population of particles of the same particle size. The pelleted EVs are resuspended in PBS and used in downstream assays.

Although this method is generally established as one of the methods for EV isolation, it poses some concerns that need to be addressed. Forcing EV passage through filters used to pre-concentrate the sample may lead to EV deformation and eventual rapture into smaller particles (30). To avoid this, it has been suggested that size exclusion chromatography should be performed by gravity or with the application of the smallest possible force $(23,30)$. Moreover, the selection of the appropriate gel type is crucial to the recovery of EVs, rather than proteins or lipoproteins. Additionally, the short isolation time and relatively low cost are also beneficial.

\section{Polymeric precipitation}

Although polymeric precipitation methods do not fall under the isolation method classification proposed above, it is worthwhile to mention them as an interesting and promising alternative. The concept behind this method is the formation of a meshlike polymeric web that captures EVs of a certain size, usually between $60-180 \mathrm{~nm}$, which are later pelleted at low centrifugal speeds. It can be used to obtain EVs from both cell culture medium and/or body fluid samples. What is very appealing about this EV isolation method is the fact that it is relatively quick, enables high $\mathrm{EV}$ recoveries and does not require laborious ultracentrifugation (53). A previous study showed the superiority of this method over others; however, it only assessed the RNA yield and protein purity/quantity (54); thus, its possible application in the overall EV recovery still needs to be addressed. Others have raised a concern regarding the contaminants, such as lipoproteins, that may be isolated along with the actual EVs (30). Moreover, since polymeric precipitation isolates EVs of $60-180 \mathrm{~nm}$ in size, it cannot be used for the assessment of larger EVs present in a sample. 


\section{Biological activities of EVs and associated challenges}

It is rather difficult to evaluate how the different isolation protocols affect the potential biological activities of EVs. The main reason for this is that the majority of available data concerns EVs isolated by a single method, and this mostly involves differential centrifugation/ultracentrifugation. At the same time, studies focusing on the comparison of the biological effects of EVs isolated by more than one protocol simply are unavailable, at least to the best of our knowledge. Most groups choose one isolation protocol through which EVs are obtained and their effect is then evaluated depending on the experimental design. For instance, exosomes isolated from red blood cells by ultracentrifugation are able to induce the release of pro-inflammatory cytokine in monocytes (55). Another study demonstrated that density-gradient isolated glioblastoma-derived EVs modified the phenotype of monocytic cells (56). It has also been shown that RNA transferred by macrophage-derived microvesicles isolated by ultracentrifugation is biologically active and induces macrophage differentiation (57). Although there are attempts to compare two or more EV isolation methods, the results are limited to the correlation of EV physical properties (e.g., number, size distribution, phenotype, protein and/or RNA expression), and do not show the biological effects. For example, in a study on EVs present in blood and urine, it was suggested that polymeric precipitation yielded the highest EV concentration, although CD133 and CD63 protein expression in these EVs was difficult to interpret compared to EVs obtained by other methods (58). What makes the assessment of EV biological activities even more difficult is that there is no single isolation protocol to refer to as the 'golden standard' guaranteeing not just the complete recovery of EVs (all the fractions), but also the recovery of EVs that retain their native form/shape and function. Thus, it is simply unknown as to whether the obtained EVs, using either one of the protocols discussed above, comprise the original EVs released by the parent cells. Due to these discrepancies, additional simultaneous studies on the effects of different isolation methods are warranted for the proper evaluation of EV biological activities.

\section{Conclusion}

The past decade has witnessed an extensive research in the EV field. As we learn more about EVs and their role under normal and/or pathological conditions, there is an increasing temptation of trying to use that knowledge in the treatment of certain diseases. Although elevated numbers of EVs or EVs bearing certain proteins, RNAs, miRNAs, etc. have been tagged as diagnostic and prognostic markers in a number of diseases, there still seems to be a question pending as to the validity of this information. The answer to this question begins with the establishment of firm EV isolation protocol(s) that would enable the assessment of the full spectrum of EVs present in a sample instantaneously. Available isolation protocols only in part meet this goal, as they tend to focus on the selection of a certain type(s) of EV, whether based on their size, density or marker expression. The outcomes of these approaches produce either inadequate $\mathrm{EV}$ numbers/concentration or contaminated EV population(s)/fraction(s), whose usefulness in downstream tests may be questionable. The isolation methods presented in this review comprise the current knowledge on the topic, shedding a light on the complexity of the EV 'world'.

\section{Acknowledgements}

This review was supported by the grant no. UMO-2012/07/B/ NZ6/03499 financed by the National Science Centre of Poland.

\section{References}

1. D'Souza-Schorey C and Clancy JW: Tumor-derived microvesicles: Shedding light on novel microenvironment modulators and prospective cancer biomarkers. Genes Dev 26: 1287-1299, 2012.

2. Ratajczak J, Miekus K, Kucia M, Zhang J, Reca R, Dvorak P and Ratajczak MZ: Embryonic stem cell-derived microvesicles reprogram hematopoietic progenitors: Evidence for horizontal transfer of mRNA and protein delivery. Leukemia 20: 847-856, 2006.

3. Mause SF and Weber C: Microparticles: Protagonists of a novel communication network for intercellular information exchange. Circ Res 107: 1047-1057, 2010.

4. Ratajczak J, Wysoczynski M, Hayek F, Janowska-Wieczorek A and Ratajczak MZ: Membrane-derived microvesicles: Important and underappreciated mediators of cell-to-cell communication. Leukemia 20: 1487-1495, 2006.

5. Cocucci E, Racchetti G and Meldolesi J: Shedding microvesicles: Artefacts no more. Trends Cell Biol 19: 43-51, 2009.

6. van der Pol E, Hoekstra AG, Sturk A, Otto C, van Leeuwen TG and Nieuwland R: Optical and non-optical methods for detection and characterization of microparticles and exosomes. J Thromb Haemost 8: 2596-2607, 2010.

7. Valadi H, Ekström K, Bossios A, Sjöstrand M, Lee JJ and Lötvall JO: Exosome-mediated transfer of mRNAs and microRNAs is a novel mechanism of genetic exchange between cells. Nat Cell Biol 9: 654-659, 2007.

8. Baj-Krzyworzeka M, Szatanek R, Weglarczyk K, Baran J, Urbanowicz B, Brański P, Ratajczak MZ and Zembala M: Tumour-derived microvesicles carry several surface determinants and mRNA of tumour cells and transfer some of these determinants to monocytes. Cancer Immunol Immunother 55: 808-818, 2006.

9. Al-Nedawi K, Meehan B, Micallef J, Lhotak V, May L, Guha A and Rak J: Intercellular transfer of the oncogenic receptor EGFRvIII by microvesicles derived from tumour cells. Nat Cell Biol 10: 619-624, 2008.

10. Vlassov AV, Magdaleno S, Setterquist R and Conrad R: Exosomes: Current knowledge of their composition, biological functions, and diagnostic and therapeutic potentials. Biochim Biophys Acta 1820: 940-948, 2012.

11. Simpson RJ, Jensen SS and Lim JW: Proteomic profiling of exosomes: Current perspectives. Proteomics 8: 4083-4099, 2008.

12. Conde-Vancells J, Rodriguez-Suarez E, Embade N, Gil D, Matthiesen R, Valle M, Elortza F, Lu SC, Mato JM and Falcon-Perez JM: Characterization and comprehensive proteome profiling of exosomes secreted by hepatocytes. J Proteome Res 7: 5157-5166, 2008.

13. Subra C, Grand D, LaulagnierK, Stella A,Lambeau G,Paillasse M, De Medina P, Monsarrat B, Perret B, Silvente-Poirot S, et al: Exosomes account for vesicle-mediated transcellular transport of activatable phospholipases and prostaglandins. J Lipid Res 51: 2105-2120, 2010.

14. Mathivanan S and Simpson RJ: ExoCarta: A compendium of exosomal proteins and RNA. Proteomics 9: 4997-5000, 2009.

15. Aharon A, Rebibo-Sabbah A, Tzoran I and Levin C: Extracellular vesicles in hematological disorders. Rambam Maimonides Med J 5: e0032, 2014.

16. Inal JM, Kosgodage U, Azam S, Stratton D, Antwi-Baffour S and Lange S: Blood/plasma secretome and microvesicles. Biochim Biophys Acta 1834: 2317-2325, 2013.

17. Piccin A, Murphy WG and Smith OP: Circulating microparticles: Pathophysiology and clinical implications. Blood Rev 21: $157-171,2007$.

18. Lynch SF and Ludlam CA: Plasma microparticles and vascular disorders. Br J Haematol 137: 36-48, 2007. 
19. Jeppesen DK, Hvam ML, Primdahl-Bengtson B, Boysen AT, Whitehead B, Dyrskjøt L, Orntoft TF, Howard KA and Ostenfeld MS: Comparative analysis of discrete exosome fractions obtained by differential centrifugation. J Extracell Vesicles 3 : $25011,2014$.

20. Théry C, Amigorena S, Raposo G and Clayton A: Isolation and characterization of exosomes from cell culture supernatants and biological fluids. Curr Protoc Cell Biol: Chapter 3: Unit 3.22, 2006. doi: 10.1002/0471143030.cb0322s30.

21. Lötvall J, Hill AF, Hochberg F, Buzás EI, Di Vizio D, Gardiner C, Gho YS, Kurochkin IV, Mathivanan S, Quesenberry P, et al: Minimal experimental requirements for definition of extracellular vesicles and their functions: A position statement from the International Society for Extracellular Vesicles. J Extracell Vesicles 3: 26913, 2014

22. Vickers KC, Palmisano BT, Shoucri BM, Shamburek RD and Remaley AT: MicroRNAs are transported in plasma and delivered to recipient cells by high-density lipoproteins. Nat Cell Biol 13: 423-433, 2011.

23. György B, Módos K, Pállinger E, Pálóczi K, Pásztói M, Misják P, Deli MA, Sipos A, Szalai A, Voszka I, et al: Detection and isolation of cell-derived microparticles are compromised by protein complexes resulting from shared biophysical parameters. Blood 117: e39-e48, 2011

24. Brinkmann V, Reichard U, Goosmann C, Fauler B, Uhlemann Y, Weiss DS, Weinrauch Y and Zychlinsky A: Neutrophil extracellular traps kill bacteria. Science 303: 1532-1535, 2004

25. Williams JC and Mackman N: MPs or ICs? Blood 117: 1101-1102, 2011.

26. Phillipson $M$ and Kubes P: The neutrophil in vascular inflammation. Nat Med 17: 1381-1390, 2011.

27. Gheldof D, Hardij J, Cecchet F, Chatelain B, Dogné JM and Mullier F: Thrombin generation assay and transmission electron microscopy: A useful combination to study tissue factor-bearing microvesicles. J Extracell Vesicles 2: 2, 2013.

28. Lacroix R, Judicone C, Mooberry M, Boucekine M, Key NS and Dignat-George F; The ISTH SSC Workshop: Standardization of pre-analytical variables in plasma microparticle determination: Results of the International Society on Thrombosis and Haemostasis SSC Collaborative workshop. J Thromb Haemost: Apr 2, 2013 (Epub ahead of print). doi: 10.1111/jth.12207.

29. Yuana Y, Bertina RM and Osanto S: Pre-analytical and analytical issues in the analysis of blood microparticles. Thromb Haemost 105: 396-408, 2011

30. Witwer KW, Buzás EI, Bemis LT, Bora A, Lässer C, Lötvall J Nolte-'t Hoen EN, Piper MG, Sivaraman S, Skog J, et al: Standardization of sample collection, isolation and analysis methods in extracellular vesicle research. J Extracell Vesicles 2: 20360, 2013

31. Ayers L, Kohler M, Harrison P, Sargent I, Dragovic R, Schaap M, Nieuwland R, Brooks SA and Ferry B: Measurement of circulating cell-derived microparticles by flow cytometry: Sources of variability within the assay. Thromb Res 127: 370-377, 2011.

32. Jayachandran M, Miller VM, Heit JA and Owen WG: Methodology for isolation, identification and characterization of microvesicles in peripheral blood. J Immunol Methods 375 : 207-214, 2012

33. Chandler WL, Yeung W and Tait JF: A new microparticle size calibration standard for use in measuring smaller microparticles using a new flow cytometer. J Thromb Haemost 9: 1216-1224, 2011.

34. Montoro-García S, Shantsila E, Tapp LD, López-Cuenca A, Romero AI, Hernández-Romero D, Orenes-Piñero E, ManzanoFernández S, Valdés M, Marín F and Lip GY: Small-size circulating microparticles in acute coronary syndromes: Relevance to fibrinolytic status, reparative markers and outcomes. Atherosclerosis 227: 313-322, 2013.

35. Sokolova V, Ludwig AK, Hornung S, Rotan O, Horn PA, Epple M and Giebel B: Characterisation of exosomes derived from human cells by nanoparticle tracking analysis and scanning electron microscopy. Colloids Surf B Biointerfaces 87: 146-150, 2011

36. Trummer A, De Rop C, Tiede A, Ganser A and Eisert R Recovery and composition of microparticles after snap-freezing depends on thawing temperature. Blood Coagul Fibrinolysis 20: 52-56, 2009

37. Zhou H, Yuen PS, Pisitkun T, Gonzales PA, Yasuda H, Dear JW, Gross P, Knepper MA and Star RA: Collection, storage, preservation, and normalization of human urinary exosomes for biomarker discovery. Kidney Int 69: 1471-1476, 2006.
38. Raposo G, Nijman HW, Stoorvogel W, Liejendekker R, Harding CV, Melief CJ and Geuze HJ: B lymphocytes secrete antigen-presenting vesicles. J Exp Med 183: 1161-1172, 1996.

39. Théry C, Ostrowski M and Segura E: Membrane vesicles as conveyors of immune responses. Nat Rev Immunol 9: 581-593, 2009.

40. Baran J, Baj-Krzyworzeka M, Weglarczyk K, Szatanek R, Zembala M, Barbasz J, Czupryna A, Szczepanik A and Zembala M: Circulating tumour-derived microvesicles in plasma of gastric cancer patients. Cancer Immunol Immunother 59: 841-850, 2010.

41. Abas L and Luschnig C: Maximum yields of microsomal-type membranes from small amounts of plant material without requiring ultracentrifugation. Anal Biochem 401: 217-227, 2010.

42. Lavoie C, Lanoix J, Kan FW and Paiement J: Cell-free assembly of rough and smooth endoplasmic reticulum. J Cell Sci 109: $1415-1425,1996$.

43. Bard MP, Hegmans JP, Hemmes A, Luider TM, Willemsen R, Severijnen LA, van Meerbeeck JP, Burgers SA, Hoogsteden HC and Lambrecht BN: Proteomic analysis of exosomes isolated from human malignant pleural effusions. Am J Respir Cell Mol Biol 31: 114-121, 2004.

44. Keller S, Ridinger J, Rupp AK, Janssen JW and Altevogt P Body fluid derived exosomes as a novel template for clinical diagnostics. J Transl Med 9: 86, 2011.

45. Poliakov A, Spilman M, Dokland T, Amling CL and Mobley JA: Structural heterogeneity and protein composition of exosomelike vesicles (prostasomes) in human semen. Prostate 69: 159-167, 2009.

46. Dettenhofer M and Yu XF: Highly purified human immunodeficiency virus type 1 reveals a virtual absence of $\mathrm{Vif}$ in virions. J Virol 73: 1460-1467, 1999.

47. Cantin R, Diou J, Bélanger D, Tremblay AM and Gilbert C: Discrimination between exosomes and HIV-1: Purification of both vesicles from cell-free supernatants. J Immunol Methods 338: 21-30, 2008

48. Yuana Y, Levels J, Grootemaat A, Sturk A and Nieuwland R: Co-isolation of extracellular vesicles and high-density lipoproteins using density gradient ultracentrifugation. J Extracell Vesicles 3: 3, 2014

49. Fitzner D, Schnaars M, van Rossum D, Krishnamoorthy G, Dibaj P, Bakhti M, Regen T, Hanisch UK and Simons M: Selective transfer of exosomes from oligodendrocytes to microglia by macropinocytosis. J Cell Sci 124: 447-458, 2011.

50. Müller G: Novel tools study cell type-specific exosomes microvesicles. J Bioanal Biomed 4: 46-60, 2012.

51. Taylor DD, Lyons KS and Gerçel-Taylor C: Shed membrane fragment-associated markers for endometrial and ovarian cancers. Gynecol Oncol 84: 443-448, 2002.

52. Böing AN, van der Pol E, Grootemaat AE, Coumans FA, Sturk A and Nieuwland R: Single-step isolation of extracellular vesicles by size-exclusion chromatography. J Extracell Vesicles 3: 3, 2014.

53. Alvarez ML, Khosroheidari M, Kanchi Ravi R and DiStefano JK Comparison of protein, microRNA, and mRNA yields using different methods of urinary exosome isolation for the discovery of kidney disease biomarkers. Kidney Int 82: 1024-1032, 2012.

54. Taylor DD, Zacharias W and Gerçel-Taylor C: Exosome isolation for proteomic analyses and RNA profiling. Methods Mol Biol 728: 235-246, 2011.

55. Danesh A, Inglis HC, Jackman RP, Wu S, Deng X, Muench MO, Heitman JW and Norris PJ: Exosomes from red blood cell units bind to monocytes and induce proinflammatory cytokines, boosting T-cell responses in vitro. Blood 123: 687-696, 2014.

56. de Vrij J, Maas SL, Kwappenberg KM, Schnoor R, Kleijn A, Dekker L, Luider TM, de Witte LD, Litjens M, van Strien ME, et al: Glioblastoma-derived extracellular vesicles modify the phenotype of monocytic cells. Int J Cancer: Mar 20, 2015 (Epub ahead of print). doi: 10.1002/ijc.29521.

57. Ismail N, Wang Y, Dakhlallah D, Moldovan L, Agarwal K, Batte K, Shah P, Wisler J, Eubank TD, Tridandapani S, et al: Macrophage microvesicles induce macrophage differentiation and miR-223 transfer. Blood 121: 984-995, 2013

58. Sáenz-Cuesta M, Arbelaiz A, Oregi A, Irizar H, OsorioQuerejeta I, Muñoz-Culla M, Banales JM, Falcón-Pérez JM, Olascoaga J and Otaegui D: Methods for extracellular vesicles isolation in a hospital setting. Front Immunol 6: 50, 2015. 